\title{
Super-Resolution Enhancement Method Based on Generative Adversarial Network for Integral Imaging Microscopy
}

\author{
Md. Shahinur Alam ${ }^{1}{ }^{\mathbb{D}}$, Ki-Chul Kwon ${ }^{1}{ }^{\mathbb{D}}$, Munkh-Uchral Erdenebat $^{1}$, Mohammed . Abbass $^{1}$, \\ Md. Ashraful Alam ${ }^{2}$ and Nam Kim ${ }^{1, *}$ \\ 1 Department of Computer and Communication Engineering, Chungbuk National University, Cheongju, \\ Chungbuk 28644, Korea; shahinur@chungbuk.ac.kr (M.S.A.); kwon@osp.chungbuk.ac.kr (K.-C.K.); \\ uchka@osp.chungbuk.ac.kr (M.-U.E.); myehiaa@chungbuk.ac.kr (M.Y.A.) \\ 2 Department of Computer Science and Engineering, BRAC University, Dhaka 1212, Bangladesh; \\ ashraful.alam@bracu.ac.bd \\ * Correspondence: namkim@chungbuk.ac.kr; Tel.: +82-043-261-2482
}

check for updates

Citation: Alam, M.S.; Kwon, K.-C.; Erdenebat, M.-U.; Y. Abbass, M.; Alam, M.A.; Kim, N.

Super-Resolution Enhancement Method Based on Generative Adversarial Network for Integral Imaging Microscopy. Sensors 2021, 21, 2164. https://doi.org/10.3390/ s21062164

Academic Editor: Bruno Tiribilli

Received: 22 February 2021

Accepted: 16 March 2021

Published: 19 March 2021

Publisher's Note: MDPI stays neutral with regard to jurisdictional claims in published maps and institutional affiliations.

Copyright: (c) 2021 by the authors. Licensee MDPI, Basel, Switzerland. This article is an open access article distributed under the terms and conditions of the Creative Commons Attribution (CC BY) license (https:/ / creativecommons.org/licenses/by/ $4.0 /)$.

\begin{abstract}
The integral imaging microscopy system provides a three-dimensional visualization of a microscopic object. However, it has a low-resolution problem due to the fundamental limitation of the F-number (the aperture stops) by using micro lens array (MLA) and a poor illumination environment. In this paper, a generative adversarial network (GAN)-based super-resolution algorithm is proposed to enhance the resolution where the directional view image is directly fed as input. In a GAN network, the generator regresses the high-resolution output from the low-resolution input image, whereas the discriminator distinguishes between the original and generated image. In the generator part, we use consecutive residual blocks with the content loss to retrieve the photo-realistic original image. It can restore the edges and enhance the resolution by $\times 2, \times 4$, and even $\times 8$ times without seriously hampering the image quality. The model is tested with a variety of low-resolution microscopic sample images and successfully generates high-resolution directional view images with better illumination. The quantitative analysis shows that the proposed model performs better for microscopic images than the existing algorithms.
\end{abstract}

Keywords: deep learning; generative adversarial network; integral imaging microscopy; machine intelligence; microscopy

\section{Introduction}

An optical microscope is a piece of magnification equipment for viewing and observing microscopic objects. The microscope is extensively used in many different fields, such as biomedical science, nanophysics, and medical science [1-3]. Generally, it consists of an objective lens and a tube lens. The specimen is placed under the objective lens, and the magnified object is viewed from the tube lens also known as the eyepiece. Magnification depends on the focal length of these two lenses. However, conventional two-dimensional (2D) microscopy only enhances the resolution and provides $2 \mathrm{D}$ information that cannot perceive the parallax and the depth information. It is a major problem where threedimensional (3D) information is necessary. To overcome this situation, different types of 3D microscopy such as confocal [3], stereoscopic [4], integral imaging microscopy (IIM)/light field microscopy (LFM) [2,5,6], and integral imaging holographic microscopy [7] have been proposed. Among these, IIM/LFM microscopy provides full 3D and parallax information. Jang and Javidi employed the first integral imaging technique for microscopic objects [8]. Later, Levoy et al. proposed and developed the first IIM microscopy [9]. An integral imaging system consists of a camera with an $M L A$ that generates elemental images. The main advantage of this system is that it takes a single shot, whereas $M L A$ works similar to multiple cameras. That is why the setup is simpler and the acquisition accuracy is far better in this case. Adjacent lens array generates perspective views in the principle of the 
stereoscopic imaging system. In fact, the IIM provides multiple perspective views (depends on the lens array) than the usual stereoscopic system. Since the IIM captures multiple views under a single camera, the resolution becomes small; hence, resolution enhancement is required.

Different resolution enhancement techniques for IIMs have been proposed [5,10-17]. Some of them are fully mechanical, such as synchronously moving lens arrays [13], pinhole array-based MLA intensity distribution methods [18], and time-multiplex systems combining low-resolution images [14]. It has a relationship between the lens array (LA) size with resolution. A larger LA enhances the resolution but reduces the depth of field (DOF). To optimize this tradeoff between them, the $M L A$-shifting method was proposed [10]. A mechanically controlled piezo-actuator was used to move the MLA in the vertical and horizontal directions ( $25 \mu \mathrm{m}$ per step), but $M L A$ shifting in the microscale is error-prone. To mitigate this problem, the interpolation-based intermediate view elemental image (IVEI) generation method was proposed using a graphic processing unit (GPU) [12]. IVEI is an interpolation-based technique where neighboring pixels are used to reconstruct another pixel. Later, the resolution was improved by applying the iterative bilinear interpolation method, which generates an orthographic view image (OVI) from the neighboring elemental image (EI) [19]. This is a relatively fast and efficient method; however, the main limitation is that the image quality dramatically decreases after some iterations. Recently, Kwon et al. proposed a deep learning-based resolution enhancement method for IIM [5] where multiple degradations based super-resolution algorithm is employed. It can enhance the resolution well; however, the quality decreases dramatically for the higher scaling factor. Hence, we focus on a scale-invariant image reconstruction system.

In this work, we improve the IIM resolution using a deep learning-based superresolution (SR) algorithm. The resolution of conventional microscopy is limited by the lens property; however, it is possible to enhance by using resolution enhancement methods, such as classical iterative interpolation-based methods [6,12,19] and deep learning-based methods $[5,15]$. The quality enhancement method takes place in the OVI. Since the resolution of the OVI is small, multiple time upscaling causes a low-quality output. To solve this problem, a deep learning-based SR algorithm is applied to the directional view images that scale up 2, 4, and 8 times larger than the original image by retaining the photo-realistic natural image. Due to the characteristics (poor lighting, distortion, etc.) of IIM image, the existing network cannot be applied as it is. In this study, the network is designed considering the IIM characteristics. Unlike other super-resolution algorithms, the proposed deep learning-based algorithm performs better for IIM. First, it retrieves edges and later synthesizes the color; on the other hand, existing algorithms perform this all together. Hence, the accuracy is significant.

The rest of the paper is organized as follows. In Section 2, we describe the necessary background for a better understanding of this work. The proposed super-resolution algorithm architecture and the processes are described in Section 3. The experimental setup and the quality measurement criteria are shown in Section 4. The result is discussed in Section 5 where we compared the result with different existing algorithms. Finally, the conclusion is provided in Section 6.

\section{Background of IIM and Super Resolution}

\subsection{Integral Imaging Microscopy}

IIM consists of a conventional microscope and an MLA. The basic schematic diagram of the IIM is shown in Figure 1. An infinity-corrected optical system is placed between the intermediate image plane and the specimen. The sensor captures the elemental image array (EIA) through MLA installed in front of the camera lens (CL). The OVI is reconstructed 
from the disparity information using the EIA. The object point $(x, y, z)$ is imaged on the EIA plane through the CL and elemental lens (EL) as Equation (1):

$$
\left\{\begin{array}{l}
X_{E I(i, j)}=\frac{f_{M L A} f_{C}\left(i \times P_{E L}-x\right)-f_{C} i \times P_{E L}\left(z-f_{M L A}\right)}{\left(g-f_{M L A}\right)\left(z-f_{M L A}\right)} \\
Y_{E I(i, j)}=\frac{f_{M L A} f_{C}\left(j \times P_{E L}-y\right)-f_{C} \times P_{E L}\left(z-f_{M L A}\right)}{\left(g-f_{M L A}\right)\left(z-f_{M L A}\right)}
\end{array}\right.
$$

where $f_{M L A}$ and $f_{C}$ are the focal lengths of the micro lens array and camera lens, respectively. Additionally, $P_{E L}$ represents the pitch of the elemental lens, which is the distance between one lens to another. The distance between the $M L A$ and the camera lens is defined as $g$. The lens position is denoted by $i$ and $j$. However, the disparity between the camera lens and the EL should be considered as Equation (2):

$$
\left\{\begin{aligned}
\Delta X_{I} & =\frac{f_{M L A} f_{C} P_{E L}\left(i_{2}-i_{1}\right)}{\left(g-f_{M L A}\right)\left(z-f_{M L A}\right)} \\
\Delta Y_{I} & =\frac{f_{M L A} f_{C} P_{E L}\left(j_{2}-j_{1}\right)}{\left(g-f_{M L A}\right)\left(z-f_{M L A}\right)}
\end{aligned}\right.
$$

where $i$ and $j$ are the same as in Equation (1). This is the depth information and viewpoint obtained for each image. If the number of lenses in an $M L A$ increases, the resolution is enhanced. However, there is a tradeoff between depth and resolution regarding the number of MLs. The number of directional view images depends on the resolution of EI, and the resolution of the sub-image depends on the number of EIs, as well as the ML. The generation of OVI from EIA is a little bit tricky. The first pixel of the first $E I$ generates the first pixel of the first OVI, the first pixel of the second EI generates the second pixel of the first OVI, and so on. Similarly, the last pixel of the last EI generates the last pixel of the last OVI [12].

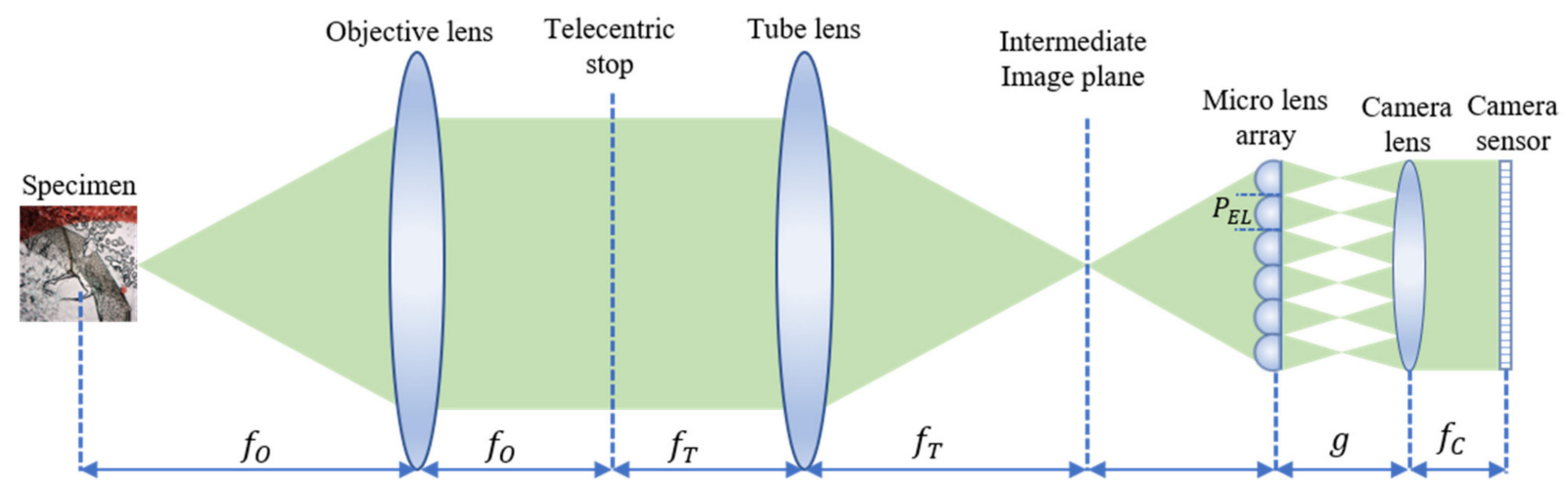

Figure 1. Schematic diagram of the integral imaging microscopy (IIM) capturing system. A specimen is placed in front of the objective lens and magnified through the tube lens; a micro lens array is placed in front of the sensor to capture the elemental image $(E I)$.

\subsection{Deep Learning-Based Super-Resolution Algorithm}

Deep learning has become popular for different applications [1,20-29]; single image SR (SISR) is one of them [27-31]. It is a very challenging problem due to the transformation of a specific low-resolution (LR) image to a high-resolution (HR) image. The main mechanism behind SISR is that it takes the original HR image and downsample multiple times to the LR image that is fed to the network to train the model. The main working principle of the SISR algorithm is shown in Figure 2. Original HR images are downsampled and convolved with noise known as kernel by the scaling factor. The LR image $y$ can be modeled as Equation (3):

$$
y=(x * n) \downarrow s+N,
$$


where $x$ is the HR part that convolved with the noise $n, \downarrow$ is the downsampling operator with factor s. $N$ (if necessary) is the independent (also known as bias) noise term. Most of the SISR algorithms work on the same concept.

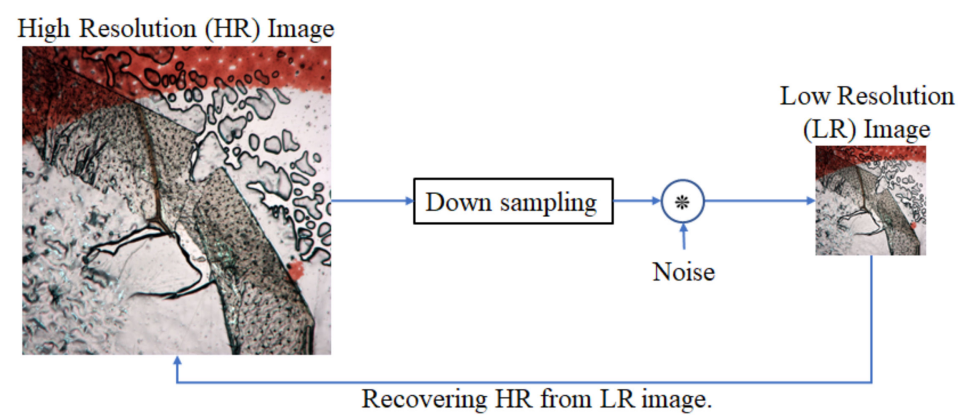

Figure 2. The basic concept of the single image super-resolution (SISR) algorithm. High-resolution images are downsampled to the corresponding low-resolution image and that low-resolution image inversely reconstructs the high-resolution image. The model is learned from the noise term during the training process.

There are different techniques for SISR algorithms; among them, the interpolationbased method is widely used [32-34]. A simple three-layer convolutional neural network (CNN)-based SR algorithm (known as SRCNN) was proposed [32]. These three nonlinear layers extract patches from the LR image map and reconstruct the HR image. Later, a pyramid structure network (LapSRN) was proposed [33]. The feature map was generated by cascading the convolutional layers; then, upscaling was performed by the cascade convolution; finally, a convolutional layer was used to predict the sub-band residuals. Recently, efficient multiple degradation-based algorithms, SRMD, was proposed [34]. It performs better in a noise-free (SRMDNF) situation. Recently, HDRN is proposed where a hierarchical dense block (HDB) is used to represent the feature module [31]. The HR image is reconstructed by the sub-pixel operation where the global fusion module is employed with HDB. However, the (first proposed in 2014 [35]) generative adversarial network (GAN)-based super-resolution algorithm is becoming popular day by day. There are different variants of GAN, such as InfoGAN [36], DCGAN [37], CycleGAN [38], and SRGAN [39]. Most of them use a rectified linear unit (ReLU) activation function [40]. It is difficult to resolve the finer texture detail for the photo-realistic natural image using general SR algorithms. However, the SRGAN can mitigate this problem using the perceptual loss function. Hence, we used a modified version of the SRGAN algorithm for resolution enhancement.

The basic concept of the GAN network is shown in Figure 3. Unlike other algorithms, it has two parts- the generator $(G)$ and the discriminator $(D)$ that train at the same time. The $G$ works based on the random variable $\mathrm{z}$ (also known as noise). Both the generated data $G(z)$ and the real data $x$ are used in $D$ to verify whether it is real or fake. It is more related to min-max rather than an optimization problem [35]. G needs to be trained simultaneously to minimize the difference between $D$ and $G$. We can define it in terms of the value function $\mathrm{V}(\mathrm{D}, \mathrm{G})$ as Equation (4):

$$
\min _{G} \max _{D} \mathrm{~V}(\mathrm{D}, \mathrm{G})=\mathbb{E}_{x \sim p_{\text {data }}(x)}[\log D(x)]+\mathbb{E}_{z \sim p_{z}(z)}[\log (1-D(G(x)))]
$$

Equation (4) maximizes the value function of D and minimizes G. With this method, the generator can learn to produce a high-quality similar image from the original one. 


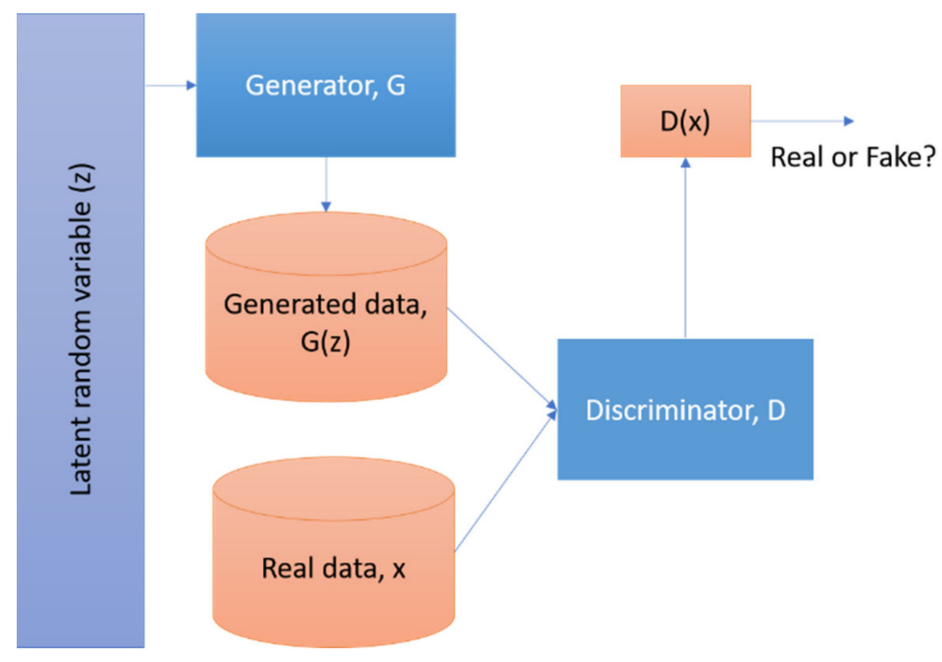

Figure 3. Schematic diagram of a basic Generative Adversarial Network. Latent variable or noise term is used in the generator part to generate the output, whereas the discriminator part compares the generated and original data to distinguish whether it is real or fake.

\section{Proposed Method for IIM Super-Resolution}

The whole process is divided into two major parts-IIM capture through MLA and resolution enhancement using the proposed GAN-based super-resolution algorithm. In the capturing process (shown in Figure 1), the specimen is placed in front of the objective lens of the microscope. The magnified image is formed in the intermediate image plane between the tube lens and the MLA. Each ML works as an individual image source that generates a perspective view image, combinedly known as $E I$. The $E I$ cannot be directly used for resolution enhancement. Therefore, the OVI is generated from the EIA, which contains the directional view information. The resolution and the number of directional view images are the same as the number of EIs and the resolution, respectively. Each directional view image was processed through the designed algorithm; the resolution was enhanced 2, 4, and 8 times and combined again for the full visualization system.

Figure 4 shows the detailed block diagram of the proposed IIM resolution enhancement system. A honeybee sample $(\sim 500 \mu \mathrm{m})$ is taken as a specimen. The EIA is captured through a camera whose resolution is $2048 \times 2048$ pixels. Due to the MLA properties, the outer side of the captured EIA contains noise; hence, a region of interest (ROI) is selected by cropping it into $1885 \times 1885$ pixels. From this selected ROI, an OVI is generated using a pixel mapping algorithm [12]. In this technique, each EI is mapped into the corresponding view image. The OVIs are fed to the designed GAN model (Figure 5) as input. The resolution of the directional view image from the OVI is individually enhanced 2, 4, and 8 times using the proposed algorithm. The directional view image generates a perspective view, which contains parallax information providing depth perception.

The main schematic diagram of the proposed algorithm is shown in Figure 5. This network is the modified version of the SRGAN algorithm. Since the scenarios are a little different in the microscopic image than the traditional one, some modifications are performed to cope with the microscopic image. Modification and network structure are described below. As mentioned earlier, the whole network has two different sections: the generator and the discriminator. 
(a) Capturing system
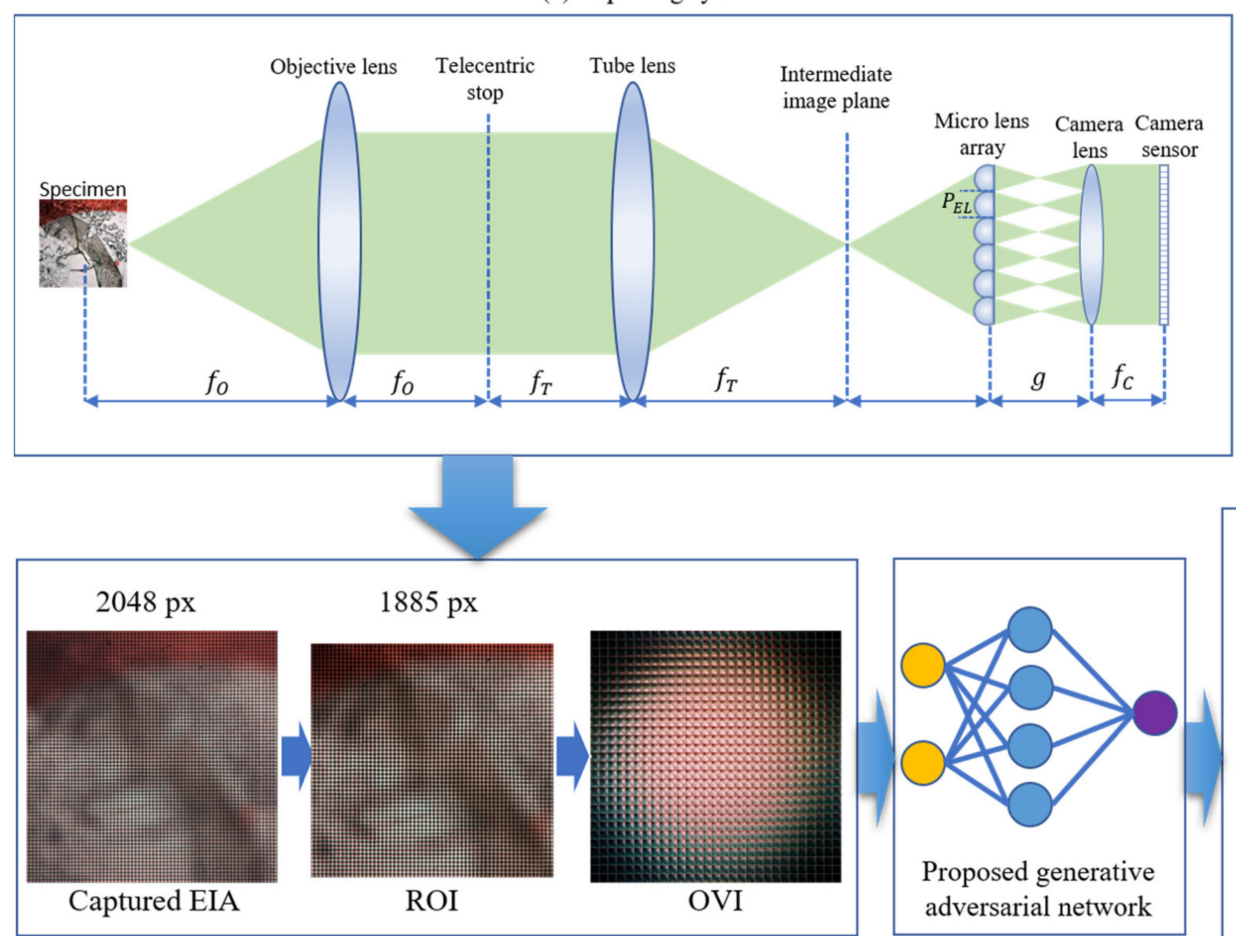

(b) Preprocessing (e) Output
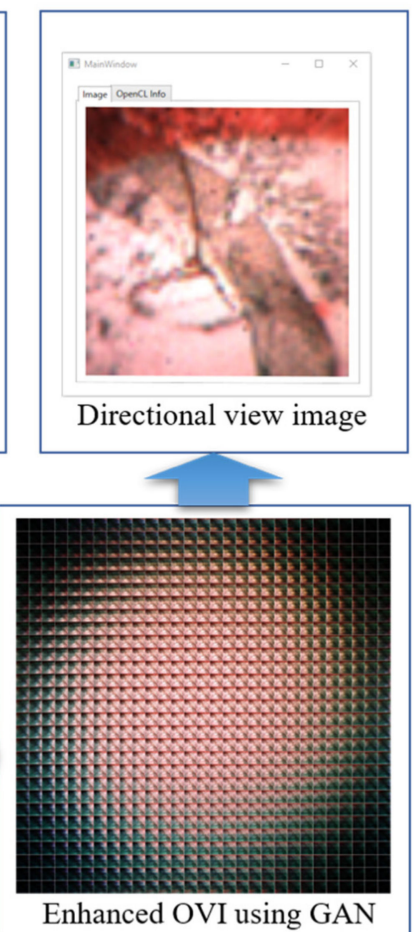

(d) Resolution enhancement

Figure 4. Block diagram of the proposed IIM resolution enhancement method. (a) An IIM capturing system where objective lens, tube lens, and micro lens array is employed to capture the EIA. (b) In the preprocessing part, the outer dark part is removed by cropping the elemental image array (EIA), and orthographic view image (OVI) is generated from the EIA using the pixel mapping algorithm. (c) A super-resolution algorithm is designed and trained using generative adversarial network (GAN). (d) The resolution of the OVI is enhanced using the SR network. (e) The resolution-enhanced directional view image is shown as an output.

\subsection{The Generator Network}

The LR image is taken as the input to the generator that passes through the convolution layer and parametric ReLU (PReLU) [41,42]. The PReLU can be defined as Equation (5):

$$
f(x)=\left\{\begin{array}{c}
x_{i}, \text { if } x_{i}>0 \\
a_{i} x_{i}, \text { if } x_{i} \leq 0
\end{array}\right.
$$

where $x_{i}$ is the input of the hidden layer on the $i$ th channel, $a_{i}$ is the coefficient of the negative part that controls the slope. The value of $a_{i}$ is learned via backpropagation during training time. When the value of $a_{i}$ is 0 , it works as a ReLU activation function. The convolution layer consists of $9 \times 9$ kernels and 64 feature maps with padding 4 . Apart from the original SRGAN, twelve residual blocks are used in the generator network. Each block consists of two convolutions followed by batch normalization (BN); after that, a PReLU is employed; the kernel size is $3 \times 3$ and the padding is 1 for both convolution layers. Due to the change of parameter in each layer for training, the learning rate reduces gradually with saturating nonlinearities. In that case, BN performs normalization for each mini-batch; hence, it allows a higher learning rate [43]. In the last residual block, there are 64 channels and 64 feature maps with kernel size $3 \times 3$ and padding 4 . To enhance the resolution of the LR input image, an upsample block is used, which consists of a convolution, pixel shuffler, and PReLU layer. A single pixel-shuffler is useful for efficient sub-pixel convolution where the super-resolution takes place in the LR instead of the HR space [44], whereas two are used in the SRGAN. It helps to retrieve the color consistency of the IIM. In this upsample block, the channel size is the same as the residual block; however, the feature map size is calculated by multiplying the channel size and the square of the up-scale factor; 
kernel size and the padding is $3 \times 3$ and 1, respectively. In the gradient-based learning method, vanishing gradient plays a vital role, sometimes the network may stop learning. To overcome this, a skip connection is established between the input and the upsampling block to prevent the gradient vanishing problem where the weight value is calculated from the previous layer. The final convolution layer contains three output channels and a $9 \times 9$ kernel size with padding 4 .
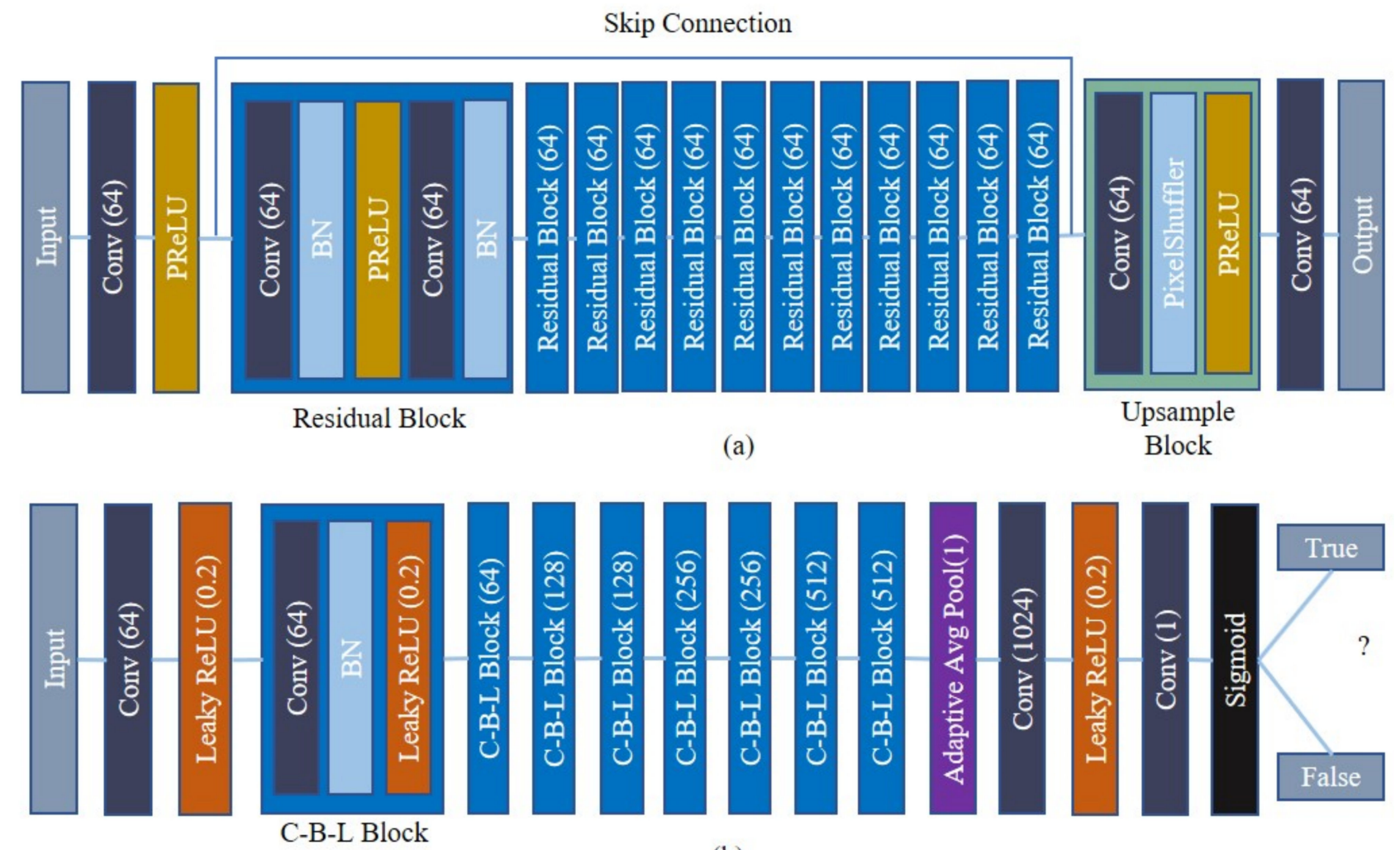

(b)

Figure 5. The architecture of the proposed GAN-based resolution enhancement algorithm: (a) generator network: the main building block of the network where noise term is used to generate the output; (b) discriminator network: discriminate between the generated and the real image.

\subsection{The Discriminator Network}

To verify the generated and original image, a discriminator network is used. After the input layer, there is a convolution (three channel and 64 feature maps) followed by a leaky ReLU layer containing a kernel size of $9 \times 9$ and padding 1 . The leaky ReLU is almost similar to the PReLU, if the value of $a_{i}$ is fixed then it becomes leaky ReLU [45], whereas the value of $a_{i}$ is variable for PReLU. In the proposed network, the value is always set to 0.2 . There are seven consecutive convolution, $\mathrm{BN}$, and leaky ReLU blocks (C-B-L Block). The C-B-L block starts with 64 channels and consecutively increases by 128, 256, and 512 . All C-B-L blocks have the same kernel size $3 \times 3$ and padding 1 . We applied stride 2 only in the odd C-B-L blocks. Adaptive average polling is used before the convolution layer. Lastly, there are two convolution layers with the output channel 1024 and 1, respectively; the kernel size is $1 \times 1$. A leaky ReLU with a constant value of 0.2 is employed between those convolution layer. A sigmoid activation function is used to discriminate whether the output is correct or not. We use the VGG feature map (pre-trained on the ImageNet dataset) to retrieve a photo-realistic image [46], whereas perceptual loss is used in the SRGAN. During the training period, a stochastic gradient-based optimizer, Adam, is used [47]. The sample data and source code of this proposed GAN network is available on GitHub (https:/ / github.com/shahinur-alam/IIM-GAN, accessed on 12 March 2021). 


\section{Experimental Setup and Quality Measurement Metrics}

In the original experimental setup shown in Figure 6, we use an Olympus BX41TF microscope with $10 \times$ magnification. An MLA composed of $100 \times 100$ lenses is used for IIM; each lens diameter and focal length are $2.4 \mathrm{~mm}$ and $125 \mu \mathrm{m}$, respectively. The image is captured using the Point Grey GS3-U3-41C6C-C, 1-inch CMOS sensor through the NIKON $20 \mathrm{~mm}$ lens. This sensor can capture 4.1 megapixels of information at $2048 \times 2048$ resolution.

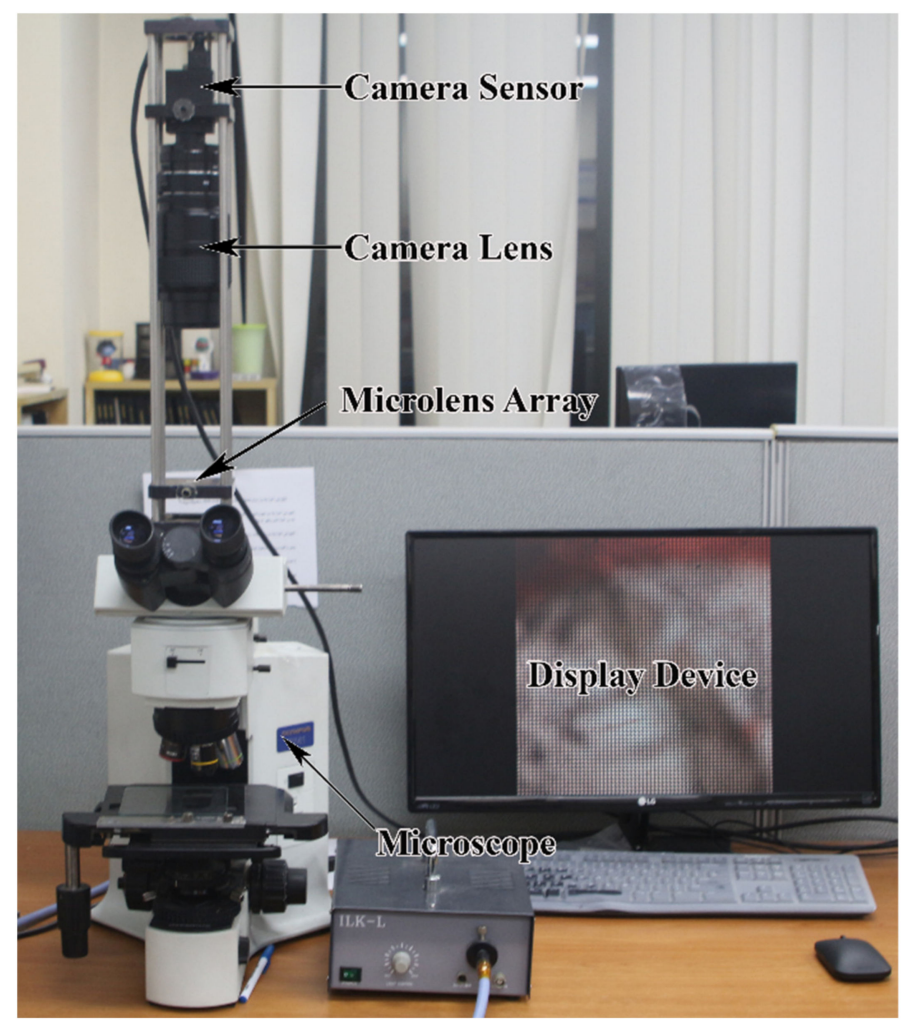

Figure 6. Experimental setup for the proposed IIM resolution enhancement system. The micro lens array (MLA) is placed between the camera sensor and the specimen, and the captured EIA is displayed in the display in real-time.

A high-configuration personal computer (PC) is used to train the model, which is composed of an Intel Core i7-9800X $3.80 \mathrm{GHz}$ processor with $128 \mathrm{~GB}$ RAM. The PC operates in a Windows 10 Pro 64-bit operating system associated with NVIDIA GeForce RTX 2080 Ti GPU. We use the Python programming language in the Anaconda environment with the PyTorch library to train and test this network. Since there is a lack of IIM dataset, the network is trained using the popular Pascal VOC2012 dataset, which contains 16,700 training and 425 validation images [48]. It has twenty classes of objects, including person, animal, vehicle, furniture, etc. Compared to other existing datasets, the VOC2012 dataset is optimal in this specific application. The training time was almost $17 \mathrm{~h}$. The generated model is tested and verified by the real microscopic specimens. The proposed network retrieves edges, that is why it can enhance the resolution of the microscopic image, though the VOC2012 dataset is a little different to the microscopic specimens.

There are different kinds of image quality measurement (IQM) techniques that are frequently used to compare the original and output images. Here, we employed the peak signal-to-noise ratio (PSNR), structural similarity index (SSIM), and power spectral density function (PSD). 


\subsection{PSNR}

PSNR is the most commonly used IQM technique since it is simple and computationally cost-efficient [49]. It is the ratio between the maximum power and noise signal. If $I(x, y)$ is the original image and $K(x, y)$ is the generated or distorted image, then PSNR is calculated as Equation (6):

$$
\mathrm{PSNR}=20 \log _{10} \frac{M A X^{2} \times M \times N}{\sum_{i=0}^{M-1} \sum_{j=0}^{N-1}[I(x, y)-K(x, y)]^{2}}
$$

where $M A X$ is the peak signal power. For an 8-bit general image, the $M A X$ value is $255 . M$ and $N$ is the resolution of the image. The instantaneous pixel value is denoted by $i$ and $j$ for the width and height of the image, respectively. PSNR has obvious physical meaning in terms of optimization. Since it uses the mean square method, the PSNR value is always nonnegative in the decibel unit.

\subsection{SSIM}

SSIM calculates the image similarity focusing on the human visual system (HVS). Unlike PSNR, SSIM not only considers the absolute error but also focuses on structural information [50]. SSIM considers the structure (s), luminance $(l)$, and the contrast $(c)$ :

$$
\begin{gathered}
s(x, y)=\frac{\sigma_{x y}+c_{3}}{\sigma_{x} \sigma_{y}+c_{3}} \\
l(x, y)=\frac{2 \mu_{x} \mu_{y}+c_{1}}{\mu_{x}^{2}+\mu_{y}^{2}+c_{1}} \\
c(x, y)=\frac{2 \sigma_{x} \sigma_{y}+c_{2}}{\sigma_{x}^{2}+\sigma_{y}^{2}+c_{2}}
\end{gathered}
$$

where $\mu_{x}, \mu_{y}, \sigma_{x}, \sigma_{y}$ are the average and variance of $x_{i}, y_{i}$, respectively. The SSIM is calculated after combining Equations (7)-(9) as:

$$
\operatorname{SSIM}(x, y)=[l(x, y)]^{\alpha}[c(x, y)]^{\beta}[s(x, y)]^{\gamma}
$$

Equation (10) calculates the final SSIM value for two signals as well as images; $\alpha$, $\beta$, and $\gamma$ are positive values that represent the magnitude of those three components. Magnitude is the user-defined value.

\section{3. $P S D$}

The power spectral density function is one kind of no-reference image quality assessment technique [50]. The power spectrum of a signal represents the power into the frequency of that signal. The PSD is calculated using the 2D Fourier transform as Equation (11):

$$
\mathrm{PSD}=\log _{10}|\digamma[x(t)]|^{2}
$$

where $x(t)$ is a time-series signal. However, Equation (11) provides continuous spectral information. To quantify the PSD value, a mean value is calculated from each spectral power.

\section{Results and Discussion of the Proposed Resolution Enhancement Method}

In this research, five different kinds of microscopic specimens (honeybee, Zea Mays (Z. Mays), hydra, chip resistor, and printed circuit board (PCB)) are used. The specimens are collected by an IIM composed of a traditional microscope and MLA. All specimens are shown in Figure 7. The PSNR, SSIM, and PSD are used to evaluate the enhanced image quality. The visual results are shown in Figures 8 and 9 for $\times 2$ and $\times 4$ upscaling of different specimens, respectively. It is observed from these figures that the output of the proposed algorithm is almost similar to the original image. Only a single OVI 
from each specimen is displayed here for better understanding. Tables 1 and 2 show the PSNR, SSIM, and PSD comparison for $\times 2$ and $\times 4$ upscaling for LapSRN [33], SRCNN [32], SRMD [34], SRMDNF [34], and SRGAN [39]. The highest obtained result is shown in boldface (Tables 1 and 3). There are two upsampling and downsampling mechanisms for SRMD and SRMDNF, and it is shown that the PSNR, SSIM, and PSD values are higher in most of the cases for bicubic interpolation than the general method.

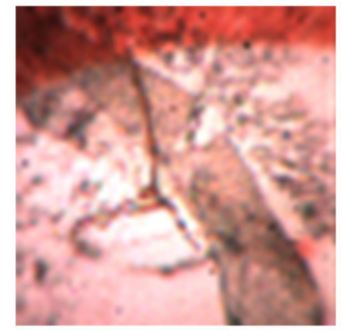

(a)

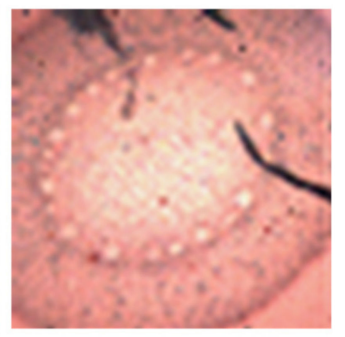

(b)

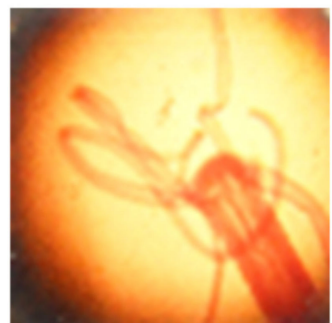

(c)

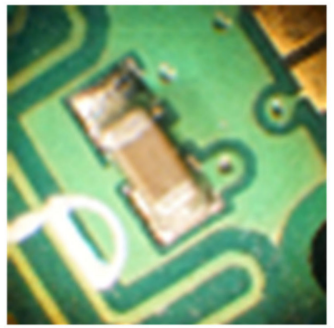

(d)

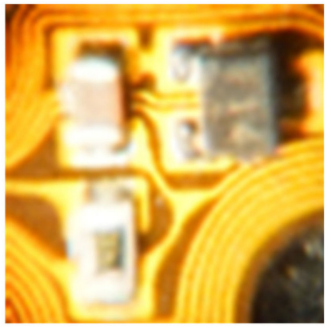

(e)

Figure 7. Different types of specimen: (a) honeybee, (b) Z. Mays, (c) hydra, (d) chip, and (e) printed circuit board (PCB).

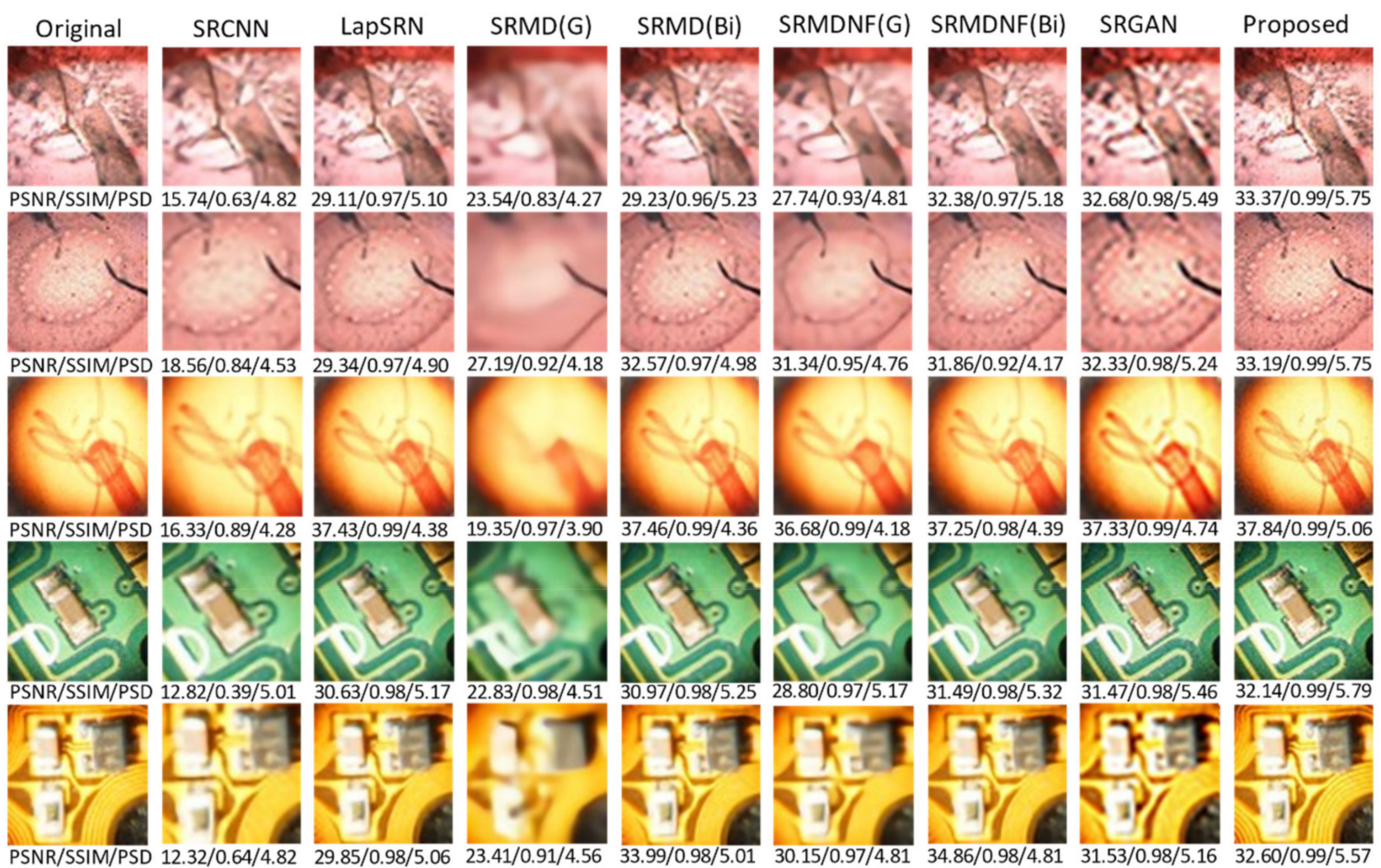

Figure 8. $\times 2$ upscaling comparison. The SRCNN, LapSRN, SRMD, SRMDNF, and SRGAN algorithms are compared with the proposed method. Most of the cases, the proposed method performs better than others, and the super-resolved image is almost indistinguishable from the original. 


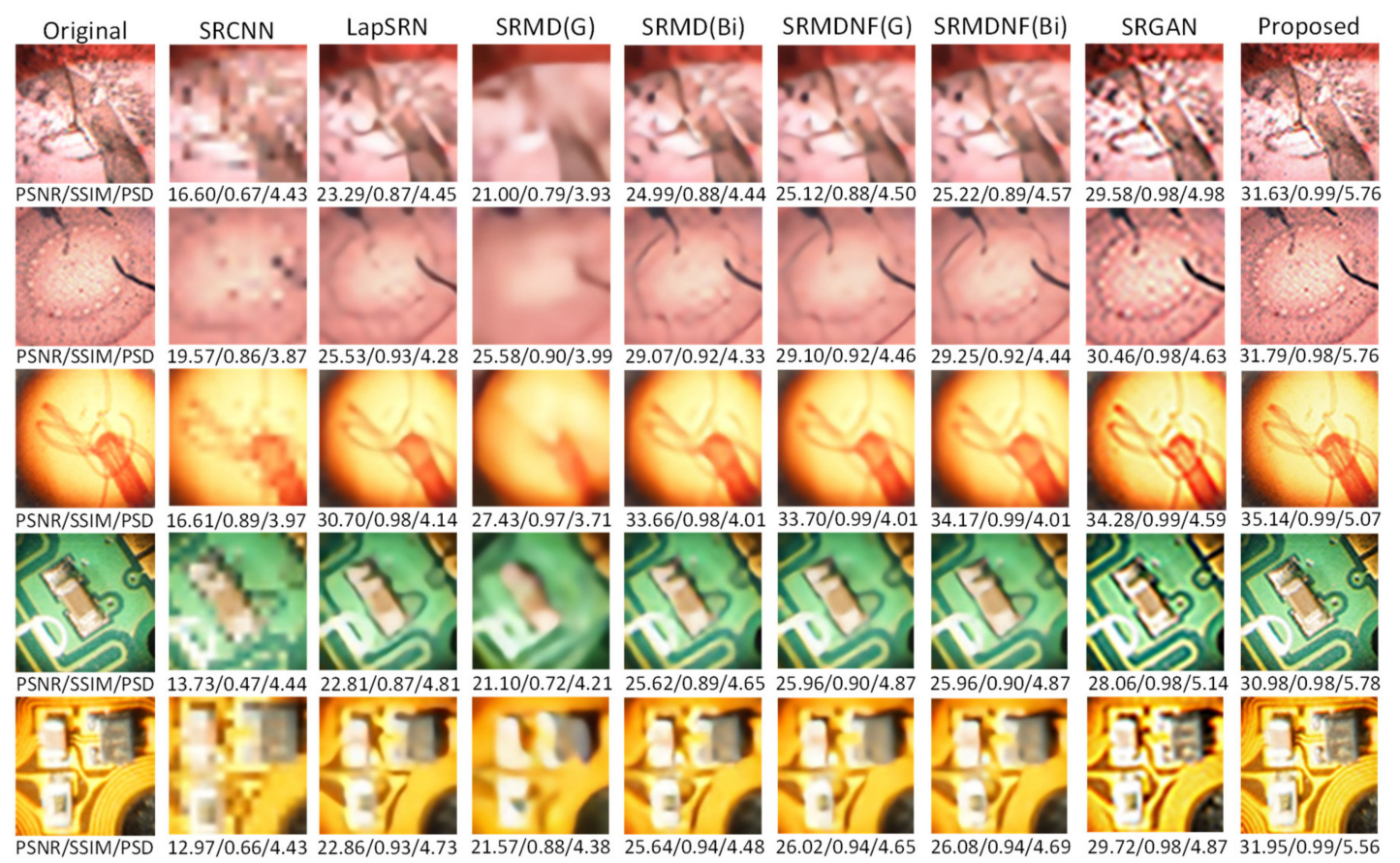

Figure 9. $\times 4$ upscaling comparison. The SRCNN, LapSRN, SRMD, SRMDNF, and SRGAN algorithm are compared with the proposed method. In all cases, the proposed method performs better than others, and the super-resolved image is almost indistinguishable from the original.

Table 1. PSNR, SSIM, and PSD comparison of the super resolved OVI $(\times 2)$ using different algorithms. Most of the cases the proposed method performs better except the PSNR of the PCB specimen. However, the other parameters are still better.

\begin{tabular}{|c|c|c|c|c|c|c|}
\hline & & Honeybee & Z. Mays & Hydra & Chip & РСВ \\
\hline \multirow{3}{*}{ SRCNN } & PSNR & 15.74 & 18.56 & 16.33 & 12.82 & 12.32 \\
\hline & SSIM & 0.63 & 0.84 & 0.89 & 0.39 & 0.64 \\
\hline & PSD & 4.82 & 4.53 & 4.28 & 5.01 & 4.82 \\
\hline \multirow{3}{*}{ LapSRN } & PSNR & 29.11 & 29.34 & 37.43 & 30.63 & 29.85 \\
\hline & SSIM & 0.97 & 0.97 & 0.99 & 0.98 & 0.98 \\
\hline & PSD & 5.10 & 4.90 & 4.38 & 5.17 & 5.06 \\
\hline \multirow{3}{*}{$\begin{array}{l}\text { SRMD } \\
\text { (general) }\end{array}$} & PSNR & 23.54 & 27.19 & 19.35 & 22.83 & 23.41 \\
\hline & SSIM & 0.83 & 0.92 & 0.97 & 0.98 & 0.91 \\
\hline & PSD & 4.27 & 4.18 & 3.90 & 4.51 & 4.56 \\
\hline \multirow{3}{*}{$\begin{array}{l}\text { SRMD } \\
\text { (bicubic) }\end{array}$} & PSNR & 29.23 & 32.57 & 37.46 & 30.97 & 33.99 \\
\hline & SSIM & 0.96 & 0.97 & 0.99 & 0.98 & 0.98 \\
\hline & PSD & 5.23 & 4.98 & 4.36 & 5.25 & 5.01 \\
\hline \multirow{3}{*}{$\begin{array}{l}\text { SRMDNF } \\
\text { (general) }\end{array}$} & PSNR & 27.74 & 31.34 & 36.68 & 28.80 & 30.15 \\
\hline & SSIM & 0.93 & 0.95 & 0.99 & 0.97 & 0.97 \\
\hline & PSD & 4.81 & 4.76 & 4.18 & 5.17 & 4.81 \\
\hline \multirow{3}{*}{$\begin{array}{l}\text { SRMDNF } \\
\text { (bicubic) }\end{array}$} & PSNR & 32.38 & 31.86 & 37.25 & 31.49 & 34.86 \\
\hline & SSIM & 0.97 & 0.92 & 0.98 & 0.98 & 0.98 \\
\hline & PSD & 5.18 & 4.17 & 4.39 & 5.32 & 4.81 \\
\hline \multirow{3}{*}{ SRGAN } & PSNR & 32.68 & 32.33 & 37.33 & 31.47 & 31.53 \\
\hline & SSIM & 0.98 & 0.98 & 0.99 & 0.98 & 0.98 \\
\hline & PSD & 5.49 & 5.24 & 4.74 & 5.46 & 5.16 \\
\hline \multirow{3}{*}{ Proposed } & PSNR & 33.37 & 33.19 & 37.84 & 32.14 & 32.60 \\
\hline & SSIM & 0.99 & 0.99 & 0.99 & 0.99 & 0.99 \\
\hline & PSD & 5.75 & 5.75 & 5.06 & 5.79 & 5.57 \\
\hline
\end{tabular}


Table 2. PSNR, SSIM, and PSD comparison of the super resolved OVI $(\times 4)$ using different algorithms. The proposed algorithm performs better in all cases.

\begin{tabular}{|c|c|c|c|c|c|c|}
\hline & & Honeybee & Z. Mays & Hydra & Chip & PCB \\
\hline \multirow{3}{*}{ SRCNN } & PSNR & 16.60 & 19.57 & 16.61 & 13.73 & 12.97 \\
\hline & SSIM & 0.67 & 0.86 & 0.89 & 0.47 & 0.66 \\
\hline & PSD & 4.43 & 3.87 & 3.97 & 4.44 & 4.43 \\
\hline \multirow{3}{*}{ LapSRN } & PSNR & 23.29 & 25.53 & 30.70 & 22.81 & 22.86 \\
\hline & SSIM & 0.87 & 0.93 & 0.98 & 0.87 & 0.93 \\
\hline & PSD & 4.45 & 4.28 & 4.14 & 4.81 & 4.73 \\
\hline \multirow{3}{*}{$\begin{array}{c}\text { SRMD } \\
\text { (general) }\end{array}$} & PSNR & 21.00 & 25.58 & 27.43 & 21.10 & 21.57 \\
\hline & SSIM & 0.79 & 0.90 & 0.97 & 0.72 & 0.88 \\
\hline & PSD & 3.93 & 3.99 & 3.71 & 4.21 & 4.38 \\
\hline \multirow{3}{*}{$\begin{array}{l}\text { SRMD } \\
\text { (bicubic) }\end{array}$} & PSNR & 24.99 & 29.07 & 33.66 & 25.62 & 25.64 \\
\hline & SSIM & 0.88 & 0.92 & 0.98 & 0.89 & 0.94 \\
\hline & PSD & 4.44 & 4.33 & 4.01 & 4.65 & 4.48 \\
\hline \multirow{3}{*}{$\begin{array}{l}\text { SRMDNF } \\
\text { (general) }\end{array}$} & PSNR & 25.12 & 29.10 & 33.70 & 25.96 & 26.02 \\
\hline & SSIM & 0.88 & 0.92 & 0.99 & 0.90 & 0.94 \\
\hline & PSD & 4.50 & 4.46 & 4.01 & 4.87 & 4.65 \\
\hline \multirow{3}{*}{$\begin{array}{c}\text { SRMDNF } \\
\text { (bicubic) }\end{array}$} & PSNR & 25.22 & 29.25 & 34.17 & 25.96 & 26.08 \\
\hline & SSIM & 0.89 & 0.92 & 0.99 & 0.90 & 0.94 \\
\hline & PSD & 4.57 & 4.44 & 4.01 & 4.87 & 4.69 \\
\hline \multirow{3}{*}{ SRGAN } & PSNR & 29.58 & 30.46 & 34.28 & 28.06 & 29.72 \\
\hline & SSIM & 0.98 & 0.98 & 0.99 & 0.98 & 0.98 \\
\hline & PSD & 4.98 & 4.63 & 4.59 & 5.14 & 4.87 \\
\hline \multirow{3}{*}{ Proposed } & PSNR & 31.63 & 31.79 & 35.14 & 30.98 & 31.95 \\
\hline & SSIM & 0.99 & 0.98 & 0.99 & 0.98 & 0.99 \\
\hline & PSD & 5.76 & 5.76 & 5.07 & 5.78 & 5.56 \\
\hline
\end{tabular}

Table 3. $\times 8$ upscaling directional view image comparison using the proposed algorithm.

\begin{tabular}{cccccc}
\hline & Honeybee & Z. Mays & Hydra & Chip & PCB \\
\hline PSNR & 31.71 & 31.89 & 35.59 & 30.48 & 31.81 \\
SSIM & 0.98 & 0.98 & 0.99 & 0.99 & 0.99 \\
PSD & 5.74 & 5.74 & 5.04 & 5.79 & 5.18 \\
\hline
\end{tabular}

From the table, in most cases, the PSNR, SSIM, and PSD values are higher for the proposed algorithm, which verifies that the method is highly suitable for microscopic specimens. There is only one case found for the PCB $\times 2$ upscaling factor where the PSNR value is higher for the SRMDNF bicubic interpolation technique. This is because of the algorithm properties and PSNR calculation technique. SRMDNF enhances the image quality without seriously considering the edges. If we take a closer look at Figure 7 and compare the PCB specimen with the SRMDNF and proposed algorithm, there is a huge edge difference. The microwires are clearly visible for the proposed algorithm but not in the SRMDNF (it seems like a single wire stripe). Since the PSNR considers the mean square error and the output is brighter for SRMDNF, it provides a higher PSNR value. However, the SSIM and PSD values are higher for the method applied here. It is a considerable flaw that most of the algorithm does not support (possibly very noisy and low-quality output) more than $\times 2$ or $\times 4$ upscaling except SRGAN, which is a great advantage for this algorithm [51]. The $\times 8$ upscaling results for the different specimens are shown in Table 3 . The results are reasonable. It is observed in Tables 1-3 that the PSNR, SSIM, and PSD values are almost identical to different quality measurement techniques, which verifies that the proposed method can retrieve a good quality image for even an $\times 8$ upscaling factor. The comparison between the PSNR, SSIM, and PSD values for different upscaling factors 
are shown in Figures 10-12, respectively. The quality and scaling factors are inversely proportional. The higher the scaling factor, the less image quality is. The PSNR values for honeybee, Z. Mays, hydra, chip, and PCB are (33.57, 33.19, 37.84, 32.14, 32.60), (31.63, $31.79,35.14,30.98,31.95),(31.71,31.89,35.59,30.48,31.81)$ for $\times 2, \times 4$, and $\times 8$ upscaling, respectively. Figure 10 shows that the PSNR value is always higher and relatively lower for the $\times 2$ and $\times 8$ upscaling, respectively. The structure of the generated images is also better for $\times 2$ upscaling (shown in Figure 11), though it is very similar to others. The SSIM values for honeybee, Z. Mays, hydra, chip, and PCB are $(0.99,0.99,0.99,0.99,0.99),(0.99$, $0.98,0.99,0.98,0.99),(0.98,0.98,0.99,0.99,0.99)$ for $\times 2, \times 4$, and $\times 8$ upscaling, respectively. The PSD values are loosely related because they depend on the specimen. Brightness and sharpness are completely different from each other. The PSD values for the honeybee, $\mathrm{Z}$. Mays, hydra, chip, and PCB are $(5.75,5.75,5.06,5.79,5.57),(5.76,5.76,5.07,5.78,5.56)$, $(5.74,5.74,5.04,5.79,5.18)$ for $\times 2, \times 4$, and $\times 8$ upscaling, respectively. PSD values are calculated in the decibel (dB) unit. It is shown in Figure 12 that the PSD values vary across different specimens. The quantitative results show that the output image is good enough to perceive a better microscopic view. However, the adversarial network produces some additional noises.

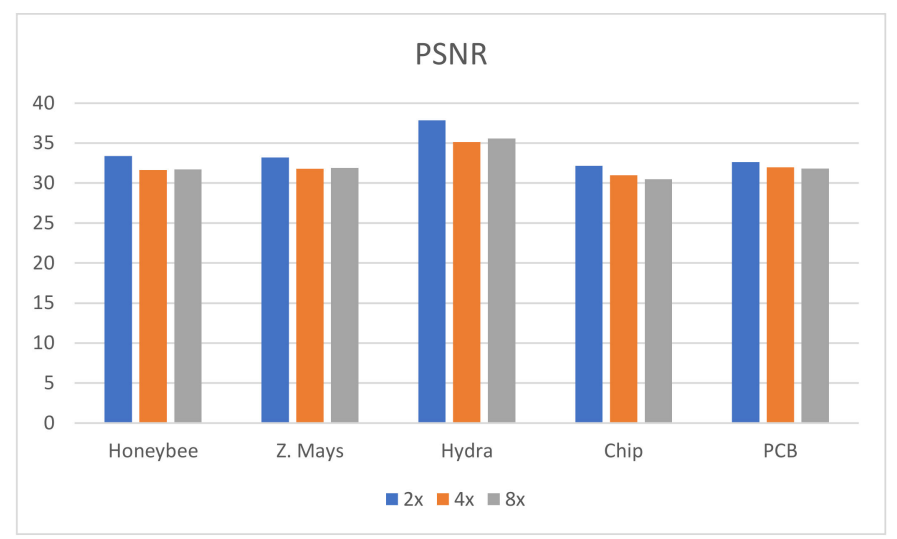

Figure 10. PSNR comparison between the $\times 2, \times 4$, and $\times 8$ upscaling factors. The maximum value found for the $\times 2$; however, compared to $\times 8$, the difference is not more than 2 .

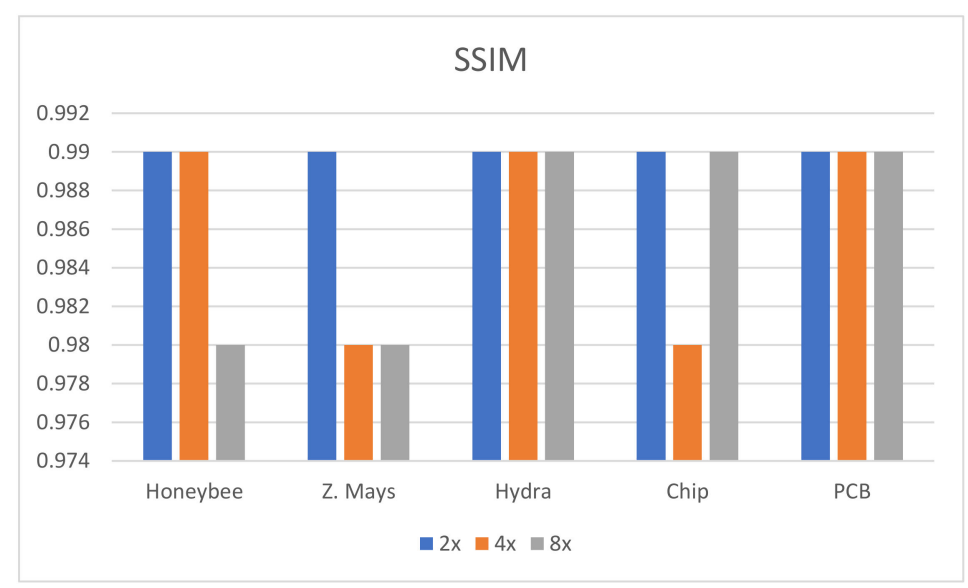

Figure 11. SSIM comparison between the $\times 2, \times 4$, and $\times 8$ upscaling factors. The best result is found for $\times 2$ upscaling, which means the output and original image is more similar. 


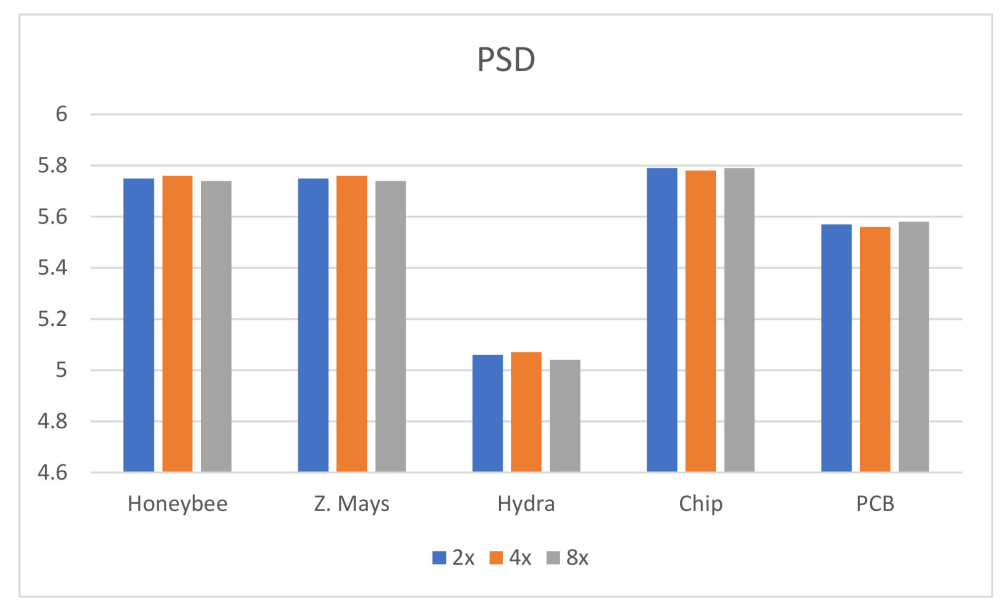

Figure 12. PSD comparison between the $\times 2, \times 4$, and $\times 8$ upscaling factors. The PSD value varies across different specimen. However, in most of the cases, the $\times 2$ upscaling performs better.

Due to the multiple ranges of the upscaling factor, the proposed method does not require the generation of any iterative interpolation-based intermediate view image [5]; in fact, the generation time dramatically increases after each iteration. However, the proposed method reduces the calculation complexity by retaining the image quality. The proposed algorithm takes, on average, $0.025 \mathrm{~s}$ to generate a one-directional view image. The training and testing times are calculated using the PyTorch default function.

\section{Conclusions}

In this paper, a useful and efficient deep learning-based resolution enhancement method for IIMs is presented. The proposed adversarial-based network efficiently handles photo-realistic images and reconstructs images similar to the original one. The EIA is captured through a camera sensor attached to the lens array and a 2D microscope. Then, the OVI is generated from the EIA according to the mapping algorithm. OVI contains multiple directional view images that generate $3 \mathrm{D}$ perception to the observer. The directional view images are directly used to feed the SR algorithm. As a result, we can obtain a high-quality resolution enhanced image.

The quantitative analysis of the PSD, SSIM, and PSNR shows that the proposed method outperforms all state-of-the-art algorithms. This algorithm requires a very short time to generate a single-view image. Additionally, it is shown that the PSNR, SSIM, and PSD values are almost identical for $\times 2, \times 4$, and $\times 8$ upscaling factors, which is a great advantage for the proposed system. Furthermore, it indicates that the enhanced image quality rarely depends on the scaling factor. In future work, getting better resolutionenhanced images and faster generation will be the main focus of this research. However, different noise suppression techniques will be applied in future work, and the state-of-theart deep learning algorithms will be compared with our improved deep learning model. Another important thing is that the IIM dataset is not adequate to date. In future work, we will also focus on making datasets for the deep learning network.

Author Contributions: Conceptualization, M.S.A. and K.-C.K.; data curation, M.S.A. and M.-U.E.; formal analysis, M.S.A. and N.K.; funding acquisition, N.K.; investigation, M.S.A. and K.-C.K.; methodology, M.S.A.; project administration, N.K.; resources, M.S.A.; software, M.S.A. and M.Y.A.; supervision, N.K.; validation, M.S.A.; visualization, M.S.A.; writing-original draft, M.S.A.; writingreview and editing, M.S.A., K.-C.K., M.-U.E., M.Y.A., M.A.A. and N.K. All authors have read and agreed to the published version of the manuscript.

Funding: This research was supported by the National Research Foundation of Korea (NRF) grant funded by the Korea government (No. NRF-2018R1D1A3B07044041 and No. NRF-2020R1A2C1101258) and Information Technology Research Center support program (IITP-2020-0-01462), supervised by the IITP (Institute for Information and communications Technology Promotion). 
Institutional Review Board Statement: Not applicable.

Informed Consent Statement: Not applicable.

Data Availability Statement: Publicly available data were analyzed in this study. This data can be found here: [https://github.com/shahinur-alam/IIM-GAN, accessed on 12 March 2021].

Acknowledgments: We want to thank all our colleagues who have helped us to complete the manuscript and supported both technically and mentally.

Conflicts of Interest: The authors declare no conflict of interest.

$\begin{array}{ll}\text { Abbreviations } \\ \text { C-B-L } & \text { Convolution, batch normalization, leaky ReLU } \\ \text { CL } & \text { Camera lens } \\ \text { DOF } & \text { Depth of field } \\ \text { EI } & \text { Elemental image } \\ \text { EIA } & \text { Elemental image array } \\ \text { EL } & \text { Elemental lens } \\ \text { GAN } & \text { Generative adversarial network } \\ \text { GPU } & \text { Graphic processing unit } \\ \text { HR } & \text { High resolution } \\ \text { HVS } & \text { Human visual system } \\ \text { IIM } & \text { Integral imaging microscopy } \\ \text { IQM } & \text { Image quality measurement } \\ \text { IVEI } & \text { Intermediate view elemental image } \\ \text { LA } & \text { Lens array } \\ \text { LFM } & \text { Light field microscopy } \\ \text { LR } & \text { Low resolution } \\ \text { MLA } & \text { Micro lens array } \\ \text { OVI } & \text { Orthographic view image } \\ \text { PCB } & \text { Printed circuit board } \\ \text { PSD } & \text { power spectral density } \\ \text { PSNR } & \text { Peak signal-to-noise ratio } \\ \text { ReLU } & \text { Rectified linear unit } \\ \text { PReLU } & \text { Parametric rectified linear unit } \\ \text { SISR } & \text { Single image super-resolution } \\ \text { SR } & \text { Super resolution } \\ \text { SSIM } & \text { Structure similarity index } \\ & \end{array}$

\section{References}

1. Belthangady, C.; Royer, L.A. Applications, promises, and pitfalls of deep learning for fluorescence image reconstruction. Nat. Methods 2019, 16, 1215-1225. [CrossRef]

2. Palmieri, L.; Scrofani, G.; Incardona, N.; Saavedra, G.; Martínez-Corral, M.; Koch, R. Robust Depth Estimation for Light Field Microscopy. Sensors 2019, 19, 500. [CrossRef] [PubMed]

3. Schlafer, S.; Meyer, R.L. Confocal microscopy imaging of the biofilm matrix. J. Microbiol. Methods 2017, 138, 50-59. [CrossRef] [PubMed]

4. Wu, Y.; Rivenson, Y.; Wang, H.; Luo, Y.; Ben-David, E.; Bentolila, L.A.; Pritz, C.; Ozcan, A. Three-dimensional virtual refocusing of fluorescence microscopy images using deep learning. Nat. Methods 2019, 16, 1323-1331. [CrossRef] [PubMed]

5. Kwon, K.-C.; Kwon, K.H.; Erdenebat, M.-U.; Piao, Y.-L.; Lim, Y.-T.; Kim, M.Y.; Kim, N. Resolution-Enhancement for an Integral Imaging Microscopy Using Deep Learning. IEEE Photonics J. 2019, 11, 1-12. [CrossRef]

6. Kim, J.; Jung, J.-H.; Jeong, Y.; Hong, K.; Lee, B. Real-time integral imaging system for light field microscopy. Opt. Express 2014, 22, 10210-10220. [CrossRef] [PubMed]

7. Kim, N.; Alam, M.A.; Bang, L.T.; Phan, A.H.; Piao, M.L.; Erdenebat, M.U. Advances in the light field displays based on integral imaging and holographic techniques (Invited Paper). Chin. Opt. Lett. 2014, 12, 060005. [CrossRef]

8. Jang, J.-S.; Javidi, B. Three-dimensional integral imaging of micro-objects. Opt. Lett. 2004, 29, 1230. [CrossRef]

9. Levoy, M.; Ng, R.; Adams, A.; Footer, M.; Horowitz, M. Light field microscopy. In Proceedings of the ACM SIGGRAPH 2006 Papers, SIGGRAPH'06, Boston, MA, USA, 30 July-3 August 2006; ACM Press: New York, NY, USA, 2006; pp. $924-934$. 
10. Lim, Y.-T.; Park, J.-H.; Kwon, K.-C.; Kim, N. Resolution-enhanced integral imaging microscopy that uses lens array shifting. Opt. Express 2009, 17, 19253. [CrossRef]

11. Kwon, K.-C.; Erdenebat, M.-U.; Lim, Y.-T.; Joo, K.-I.; Park, M.-K.; Park, H.; Jeong, J.-R.; Kim, H.-R.; Kim, N. Enhancement of the depth-of-field of integral imaging microscope by using switchable bifocal liquid-crystalline polymer micro lens array. Opt. Express 2017, 25, 30503. [CrossRef]

12. Kwon, K.-C.; Jeong, J.-S.; Erdenebat, M.-U.; Lim, Y.-T.; Yoo, K.-H.; Kim, N. Real-time interactive display for integral imaging microscopy. Appl. Opt. 2014, 53, 4450. [CrossRef]

13. Jang, J.-S.; Javidi, B. Improved viewing resolution of three-dimensional integral imaging by use of nonstationary micro-optics. Opt. Lett. 2002, 27, 324. [CrossRef] [PubMed]

14. Kishk, S.; Javidi, B. Improved resolution 3D object sensing and recognition using time multiplexed computational integral imaging. Opt. Express 2003, 11, 3528. [CrossRef]

15. Rivenson, Y.; Göröcs, Z.; Günaydin, H.; Zhang, Y.; Wang, H.; Ozcan, A. Deep learning microscopy. Optica 2017, 4, 1437. [CrossRef]

16. Martinez-Corral, M.; Dorado, A.; Barreiro, J.C.; Saavedra, G.; Javidi, B. Recent Advances in the Capture and Display of Macroscopic and Microscopic 3-D Scenes by Integral Imaging. Proc. IEEE 2017, 105, 825-836. [CrossRef]

17. Alam, S.; Kwon, K.-C.; Erdenebat, M.-U.; Lim, Y.-T.; Imtiaz, S.; Sufian, M.A.; Jeon, S.-H.; Kim, N. Resolution Enhancement of an Integral Imaging Microscopy Using Generative Adversarial Network. In Proceedings of the 14th Pacific Rim Conference on Lasers and Electro-Optics (CLEO PR 2020) (2020), paper C3G_4, The Optical Society, Sydney, Australia, 2-6 August 2020.

18. Erdmann, L.; Gabriel, K.J. High-resolution digital integral photography by use of a scanning microlens array. Appl. Opt. 2001, 40, 5592. [CrossRef] [PubMed]

19. Kwon, K.-C.; Jeong, J.-S.; Erdenebat, M.-U.; Piao, Y.-L.; Yoo, K.-H.; Kim, N. Resolution-enhancement for an orthographic-view image display in an integral imaging microscope system. Biomed. Opt. Express 2015, 6, 736-746. [CrossRef]

20. Kwon, H.; Yoon, H.; Park, K.-W. CAPTCHA Image Generation: Two-Step Style-Transfer Learning in Deep Neural Networks. Sensors 2020, 20, 1495. [CrossRef]

21. Kwon, H.; Yoon, H.; Park, K.W. Robust CAPTCHA image generation enhanced with adversarial example methods. IEICE Trans. Inf. Syst. 2020, 103, 879-882. [CrossRef]

22. Zhang, Q.; Yang, L.T.; Chen, Z.; Li, P. A survey on deep learning for big data. Inf. Fusion 2018, 42, 146-157. [CrossRef]

23. Litjens, G.; Kooi, T.; Bejnordi, B.E.; Setio, A.A.A.; Ciompi, F.; Ghafoorian, M.; van der Laak, J.A.W.M.; van Ginneken, B.; Sánchez, C.I. A survey on deep learning in medical image analysis. Med. Image Anal. 2017, 42, 60-88. [CrossRef]

24. Alam, M.S.; Kwon, K.-C.; Alam, M.A.; Abbass, M.Y.; Imtiaz, S.M.; Kim, N. Trajectory-Based Air-Writing Recognition Using Deep Neural Network and Depth Sensor. Sensors 2020, 20, 376. [CrossRef] [PubMed]

25. Xie, J.; Girshick, R.; Farhadi, A. Deep3D: Fully Automatic 2D-to-3D Video Conversion with Deep Convolutional Neural Networks; European Conference on Computer Vision; Springer: Cham, Switzerland, 2016.

26. Nguyen-Phuoc, T.; Li, C.; Theis, L.; Richardt, C.; Yang, Y.L. HoloGAN: Unsupervised learning of 3D representations from natural images. In Proceedings of the 2019 International Conference on Computer Vision Workshop, ICCVW 2019, Seoul, Korea, 27 October-2 November 2019; pp. 2037-2040.

27. Yang, W.; Zhang, X.; Tian, Y.; Wang, W.; Xue, J.-H.; Liao, Q. Deep Learning for Single Image Super-Resolution: A Brief Review. IEEE Trans. Multimed. 2019, 21, 3106-3121. [CrossRef]

28. Abbass, M.Y.; Kwon, K.C.; Alam, M.S.; Piao, Y.L.; Lee, K.Y.; Kim, N. Image super resolution based on residual dense CNN and guided filters. Multimed. Tools Appl. 2020, 80,1-19. [CrossRef]

29. LeCun, Y.; Bengio, Y.; Hinton, G. Deep learning. Nature 2015, 521, 436-444. [CrossRef] [PubMed]

30. Mei, Y.; Fan, Y.; Zhou, Y.; Huang, L.; Huang, T.S.; Shi, H. Image super-resolution with cross-scale non-local attention and exhaustive self-exemplars mining. In Proceedings of the IEEE Computer Society Conference on Computer Vision and Pattern Recognition, Seattle, WA, USA, 13-19 June 2020; IEEE Computer Society: Piscataway, NJ, USA, 2020; pp. 5689-5698.

31. Jiang, K.; Wang, Z.; Yi, P.; Jiang, J. Hierarchical dense recursive network for image super-resolution. Pattern Recognit. 2020, 107, 107475. [CrossRef]

32. Dong, C.; Loy, C.C.; He, K.; Tang, X. Image Super-Resolution Using Deep Convolutional Networks. IEEE Trans. Pattern Anal. Mach. Intell. 2016, 38, 295-307. [CrossRef]

33. Lai, W.-S.; Huang, J.-B.; Ahuja, N.; Yang, M.-H. Deep Laplacian Pyramid Networks for Fast and Accurate Super-Resolution. In Proceedings of the 2017 IEEE Conference on Computer Vision and Pattern Recognition (CVPR), Honolulu, HI, USA, 21-26 July 2017; IEEE: Piscataway, NJ, USA, 2017; Volume 2017, pp. 5835-5843.

34. Zhang, K.; Zuo, W.; Zhang, L. Learning a Single Convolutional Super-Resolution Network for Multiple Degradations. In Proceedings of the IEEE Computer Society Conference on Computer Vision and Pattern Recognition, Salt Lake City, UT, USA, 18-23 June 2018; IEEE: Piscataway, NJ, USA, 2018; pp. 3262-3271.

35. Goodfellow, I.J.; Pouget-Abadie, J.; Mirza, M.; Xu, B.; Warde-Farley, D.; Ozair, S.; Courville, A.; Bengio, Y. Generative Adversarial Nets. In Proceedings of the International Conference on Neural Information Processing Systems, Montreal, QC, Canada, 8-13 December 2014; pp. 2672-2680.

36. Chen, X.; Duan, Y.; Houthooft, R.; Schulman, J.; Sutskever, I.; Abbeel, P. InfoGAN: Interpretable Representation Learning by Information Maximizing Generative Adversarial Nets. arXiv 2016, arXiv:1606.03657. (preprint). 
37. Radford, A.; Metz, L.; Chintala, S. Unsupervised representation learning with deep convolutional generative adversarial networks. arXiv 2015, arXiv:1511.06434. (preprint).

38. Zhu, J.-Y.; Park, T.; Isola, P.; Efros, A.A. Unpaired Image-to-Image Translation using Cycle-Consistent Adversarial Networks. Proc. IEEE Int. Conf. Comput. Vis. 2017, 2223-2232.

39. Ledig, C.; Theis, L.; Huszar, F.; Caballero, J.; Cunningham, A.; Acosta, A.; Aitken, A.; Tejani, A.; Totz, J.; Wang, Z.; et al. PhotoRealistic Single Image Super-Resolution Using a Generative Adversarial Network. In Proceedings of the 2017 IEEE Conference on Computer Vision and Pattern Recognition (CVPR), Las Vegas, NV, USA, 26 June-1 July 2016; pp. $105-114$.

40. Nair, V.; Hinton, G.E. Rectified Linear Units Improve Restricted Boltzmann Machines. In Proceedings of the 27th International Conference on International Conference on Machine Learning, Haifa, Israel, 21-24 June 2010; Omnipress: Madison, WI, USA, 2010; pp. 807-814.

41. Xu, B.; Wang, N.; Chen, T.; Li, M. Empirical Evaluation of Rectified Activations in Convolutional Network. arXiv 2015, arXiv:1505.00853.

42. He, K.; Zhang, X.; Ren, S.; Sun, J. Delving deep into rectifiers: Surpassing human-level performance on imagenet classification. In Proceedings of the IEEE International Conference on Computer Vision, Santiago, Chile, 7-13 December 2015; Volume 2015, pp. 1026-1034.

43. Ioffe, S.; Szegedy, C. Batch normalization: Accelerating deep network training by reducing internal covariate shift. In Proceedings of the 32nd International Conference on Machine Learning, ICML 2015, Lille, France, 6-11 July 2015; International Machine Learning Society (IMLS): Baltimore, ML, USA, 2015; Volume 1, pp. 448-456.

44. Shi, W.; Caballero, J.; Huszar, F.; Totz, J.; Aitken, A.P.; Bishop, R.; Rueckert, D.; Wang, Z. Real-Time Single Image and Video SuperResolution Using an Efficient Sub-Pixel Convolutional Neural Network. In Proceedings of the IEEE Computer Society Conference on Computer Vision and Pattern Recognition, Las Vegas, NV, USA, 27-30 June 2016; IEEE Computer Society: Washington, DC, USA, 2016; Volume 2016, pp. 1874-1883.

45. Maas, A.L.; Hannun, A.Y.; Ng, A.Y. Rectifier nonlinearities improve neural network acoustic models. In Proceedings of the in ICML Workshop on Deep Learning for Audio, Speech and Language Processing, Atlanta, GA, USA, 16-21 June 2013.

46. Simonyan, K.; Zisserman, A. Very Deep Convolutional Networks for Large-Scale Image Recognition. arXiv 2014, arXiv:1409.1556.

47. Kingma, D.P.; Ba, J. Adam: A Method for Stochastic Optimization. arXiv 2014, arXiv:1412.6980.

48. The PASCAL Visual Object Classes Challenge (VOC2012). 2012. Available online: http://host.robots.ox.ac.uk/pascal/VOC/voc2 012/ (accessed on 15 June 2020).

49. Mandal, J.K.; Satapathy, S.C.; Sanyal, M.K.; Sarkar, P.P.; Mukhopadhyay, A. Analysis and Evaluation of Image Quality Metrics. Adv. Intell. Syst. Comput. 2015, 340, 369-378.

50. Wang, Z.; Bovik, A.C.; Sheikh, H.R.; Simoncelli, E.P. Image quality assessment: From error visibility to structural similarity. IEEE Trans. Image Process. 2004, 13, 600-612. [CrossRef] [PubMed]

51. Wang, Z.; Chen, J.; Hoi, S.C.H. Deep Learning for Image Super-resolution: A Survey. IEEE Trans. Pattern Anal. Mach. Intell. 2020, 1. [CrossRef] [PubMed] 